\title{
A Single-blind Randomised Trial of Intra-Articular Hyaluronic Acid, Hypertonic Saline, and Physiotherapy in Knee Osteoarthritis
}

\author{
E. Taftain ${ }^{1}$, S. Azizi ${ }^{1}$, A. Dadarkhah ${ }^{1}$, N. Maghbouli², S. Najafi' ${ }^{1}$ Z. Reza Soltani' \\ A. Khavandegar ${ }^{3}$ \\ 1 Research Center of Clinical Biomechanics and Ergonimics, Faculty of Medicine, Aja University of Medical \\ Sciences, Tehran, Iran \\ 2 Department of Physical Medicine and Rehabilitation, Faculty of Medicine, IKHC, Tehran University of Medical \\ Science, Tehran, Iran \\ 3 Student Research Committee, Alborz University of Medical Sciences, Karaj, Iran
}

\author{
CORRESPONDING AUTHOR: \\ Zahra Reza Soltani \\ Research Center of Clinical Biomechanics \\ and Ergonimics \\ Faculty of Medicine \\ Aja University of Medical Sciences \\ Etemadzadeh St \\ Western Fatemi, Tehran, Islamic Republic \\ of Iran \\ E-mail: z.rezasoltani@ajaums.ac.ir
}

DOI:

10.32098/mltj.03.2021.05

LEVEL OF EVIDENCE: 1B

\begin{abstract}
SUMMARY
Background. Knee osteoarthritis $(\mathrm{OA})$ is an age-related progressive condition that has resulted in a significant burden worldwide. In this survey, we aimed to compare intra-articular hypertonic saline (HS) and hyaluronic acid (HA) safety and efficacy in the pain and function improvement of patients suffering from knee OA. Eventually, we made a comparison with physical therapy $(\mathrm{PH})$, in a six-month follow-up.

Methods. This study is a three-arm single-blinded randomized clinical trial evaluating 130 radiographically confirmed knee osteoarthritis patients suffering from chronic knee pain. A group of participants received three weekly injections of HA (Hyal$\left.\operatorname{gan}^{\circledast}\right)$, while the other group experienced an injection of five milliliters of $5 \%$ HS and the third group engaged in ten-session physiotherapy. Patients assessed before intervention, one, three, and six months post-intervention using the visual analogue scale (VAS) for pain and Knee injury and osteoarthritis outcome score (KOOS) for function and quality of life (QOL).

Results. In this study, We did not identify any remarkable adverse effects. All KOOS subscales scores decreased in the HA and HS group significantly. A similar trend was detected in pain improvement, considering VAS. PH group experienced only pain reduction but not function improvement. Comparing two injected agents, HS group obtained better scores not only in pain and symptom reduction, but also in function and QOL improvement.

Conclusions. However, both HS and HA can be effectively used for improving function and reducing pain and unpleasant symptoms in knee OA patients, HS prolotherapy demonstrated more acceptable outcomes through six-month follow-up.
\end{abstract}

\section{KEY WORDS}

Hyaluronic acid; bypertonic; knee; osteoarthritis; prolotherapy; physical therapy modality; saline solution.

\section{INTRODUCTION}

Knee osteoarthritis $(\mathrm{OA})$ is an age-related progressive condition resulting in cartilage damage besides other joint components damage, including bone, synovium, and ligaments (1). This disease is the result of genetic, metabolic, and biomechanical factors interaction, eventually leading to pain and stiffness (2). Considering decreased function and impaired quality of life, $\mathrm{OA}$ is one of the leading causes of disabilities among industrialized and developing societies (3). Studies reported that the lifelong probability of symptomatic knee OA is $44.7 \%$, with a total cost of $11.1 \mathrm{k} €$ annually for such patients $(4,5)$. Albeit there is no existing defi- 
nite treatment for OA, several options for pain management and suppression of OA rapid progression have been developed. Pharmacologic treatments are believed to be useful in OA-related pain management; moreover, the adverse events, including gastrointestinal complications of non-steroidal anti-inflammatory drugs (NSAIDs) and cardiovascular complications of cyclooxygenase-2 (COX-2) inhibitors, are of importance for clinicians $(6,7)$.

Other conservative treatments usually include therapeutic exercises, lifestyle modification, different intra-articular injections, and supplements such as chondroitin sulfate and glucosamine $(8,9)$. Furthermore, studies approved the effectiveness of Transcutaneous electrical nerve stimulation (TENS) and therapeutic ultrasound $(10,11)$. Total knee arthroplasty seems to be an appropriate option for drug-resistant pain in severe cases.

Growing evidence supports the use of prolotherapy as a therapeutic alternative for chronic musculoskeletal pain management $(12,13)$. Irritant agents, usually hyperosmolar dextrose, are injected intra/extra-articular. Hyperpolarization of joint nerves opening potassium channels is the first suggestive mechanism (14). The second mechanism is local healing stimulation following cell degradation due to osmolality effects and the recruitment of inflammatory cascades (15).

Reviewing the literature, prolotherapy's clinical outcomes are mostly reported in chronic low back pain; besides, chronic tendinopathies and OA are highlighted $(16,17)$.

Recent systematic reviews claimed some OA patients revealed symptoms relief in the saline arm of the clinical trials and questioned the use of saline as a placebo (18-20). Studies approved that hypertonic saline increased the synthesis of NFAT5 as the transcription factor participating in osmolar regulation of anti-inflammatory molecules in joints $(21,22)$; however, existing studies regarding basic sciences supporting evidence of HS effects remained insufficient. Moreover, the protracted history of HS use as a placebo treatment supports its safety. According to a meta-analysis of evidence Level 1 studies, assessing 13 cohorts, for the evaluation of therapeutic effects of saline intra-articular injection, improvements in both pain and function through a six-month follow up were detected (20).

Hyaluronic acid (HA) as a mucopolysaccharide is naturally present in the synovial joints and could be destroyed after knee OA (23). From a mechanism point of view, HA injection in joints has both viscoelastic and anti-inflammatory effects (24). There is a large body of evidence suggesting that HA efficacy in the knee OA treatment is considerable $(25,26)$. A meta-analysis showed that HA effects last longer time (till one year) in comparison with corticosteroids (one month) (27).
Due to the cost-effectiveness and existing uncertainty about HS use, our aim in this study is to compare physical therapy $(\mathrm{PH})$, intra-articular HS, and HA safety and efficacy in pain and function improvement of patients with knee OA in a six-month follow-up.

\section{MATERIALS AND METHODS}

This study is a single-blinded randomized clinical trial with three-arm, implemented in the Physical medicine and rehabilitation (PM \& R) clinic of Emam Reza Hospital in Tehran from May 2019 to January 2020. The protocol of this study is approved by the ethics committee of AJA university of medical sciences (code: IR.AJAUMS.REC.1398.003) and registered in the Iranian Registry of Clinical Trials Database (IRCT: IRCT20190309042989N1).

\section{Sample size calculation}

The primary outcome measure of this study was pain; therefore, the sample size was calculated based on VAS score with a statistical level of significance; 0.05 and test power of 0.8 using repeated-measures ANOVA. In conclusion, 30 participants calculated for each group.

\section{Inclusion criteria}

Patients at the age of 50-80 years with symptomatic knee OA lasting for more than three months despite receiving conservative treatments (exercise and oral/topical drugs) were included in our study. The diagnosis of OA was based on the American College of Rheumatology criteria. Among patients suffering from both limbs OA, the more damaged knee was determined for the study participation. Patients with a Kellgren-Lawrance radiologic (KLS) score of 2-4 were included after obtaining written informed consent.

\section{Exclusion criteria}

Our exclusion criteria were recent history of trauma or surgery in the studied joint during the past six months, history of neuromuscular disorders, history of previous IA steroid injection into the studied knee within recent three months or HA injection within one year, use of systemic steroids (except inhaling types) within three months, severe deformity in lower limbs, and coagulopathies. We also excluded pregnant women, patients receiving immunosuppressant treatments for their underlying rheumatologic disease. Patients asked to inform the physician if they tended to use other analgesics or therapeutic techniques during the study. 


\section{Intervention}

Patients were randomly assigned to three groups; HA, HS, and $\mathrm{PH}$. Simple randomization, using a random number table, was applied as a randomized assignment method. The researcher randomly selected the number and assigned it to the specific group. For maintaining further blindness in this study, coded folders were used. One of our team members, who were aware of group assignments, kept the coded folders.

Before all injections, we used two milliliters of Lidocaine $2 \%$ solution for increasing patient cooperation in the procedure. We injected through a $23-G$ needle, with an anteromedial approach in the supine position and $90^{\circ}$ flexion of knee considering sterility. The participating physician in injecting patients was not blind to the injection material due to the specific shape of the HA syringe and did not participate in follow-up or patient assessment. The assessor physician was blind to the injected agent for each patient. Patients were prescribed an ice pack 3-4 times a day for two days after injection. Furthermore, patients were to avoid high-energy activities on the injected limb. They were also educated on lifestyle modifications and strengthening exercises (quadriceps, hip abductors, and adductors) with filling checklist, including exercise adherence.

HA was injected as a $20 \mathrm{mg} / 2 \mathrm{ml}$ solution of HA (Hyalgan ${ }^{\circledR}$, a viscous product with a molecular weight of $500-730 \mathrm{kDa}$, containing purified natural hyaluronate in sodium chloride solution; Fidia Farmaceutici S.p.A., Abano Terme, Italy) for three times. For the HS group, five milliliters of hypertonic saline solution (Hpersaline ${ }^{\circledR} 5 \%$, a concentrated Sodium Chloride solution and $1.71 \mathrm{Osmol} / \mathrm{ml}$; Shahid Ghazi pharmaceutical company, Tabriz, Iran) was used.

The physiotherapy group received infra-red (IR) from a distance of 50 centimeters for 20 minutes and transcutaneous electrical stimulation (TENS) with protocol consisting of 100-150 Hz frequency and 150-microsecond pulse duration and intensity of patient tolerance. Additionally, ultrasound was used on the affected knee with a duration of 3-5 minutes (1 Mega-Hertz, $1.5 \mathrm{Watt} / \mathrm{cm}^{2}$, and pulsed type).

\section{Outcome measurements}

The primary outcome measure was pain on walking 50 meters using a 10-level visual analogue scale (VAS).

Secondary outcome measures were the Knee injury and osteoarthritis outcome score (KOOS). The KOOS includes five domains and 42 items. This scale was used to evaluate symptoms, pain, function, daily living, sports/recreational activities, and quality of life in knee osteoarthritis patients (28). The main difference between the Western Ontario and
McMaster Universities Osteoarthritis Index (WOMAC) and the KOOS is the inclusion of two additional domains, a five-item sport and recreation function domain, and a four-item QOL domain. Also, the pain domain and stiffness domain were changed to a seven-item symptom domain with more detailed questions (29). For each domain, scores were normalized on a "0-100" scale; zero for the worst result and 100 for the best result. Patients were evaluated before and after the procedure on the first, third, and sixth months after the intervention.

\section{Data analysis}

Statistical Analyses was performed by STATA software version 14; figures and graphs were depicted by prism 5 software. P-value of less than 0.05 was considered significant. Categorical data are presented as percent, and Continuous demographic variables are presented as Mean \pm Standard deviation or Median (Interquartile range). For Further analyses, MITT, which is a subset of Full Intention-to-treat analysis (Full ITT), was used. Participants who left the study for any reason and whose data were not available were excluded; the final analysis was accomplished on existing data. The intervening effects on primary and secondary outcomes were investigated for longitudinal data using General Estimation Equation (GEE).

Furthermore, to evaluate the GEE model, the residual of the model was analyzed and had a symmetric distribution. The GEE model consists of group, time, and group-time interaction. The GEE model demonstrates the adjusted interference effects compared to the baseline value and demographic information. The correlation matrix was considered for each exchangeable outcome. For continuous outcomes, the identity link was used.

\section{RESULTS}

\section{Study adherence}

A total of 130 consecutive patients, 43 in the $\mathrm{PH}$ group, 43 in the HS group, and 44 in the HA group participated in the study. Totally 27 patients were excluded due to lack of access or not accepting concealed allocation. Three patients left the study during the follow-up. In the end, data from 100 participants used for analysis (figure 1).

All patients declared personal problems as their reason for departure, including a tendency to try other treatment options, and they denied any complications or side effects of intervention as a reason to leave. The treatment groups were approximately similar at baseline demographic and radiographic characteristics. The outcome measures (QOL, ADL, 


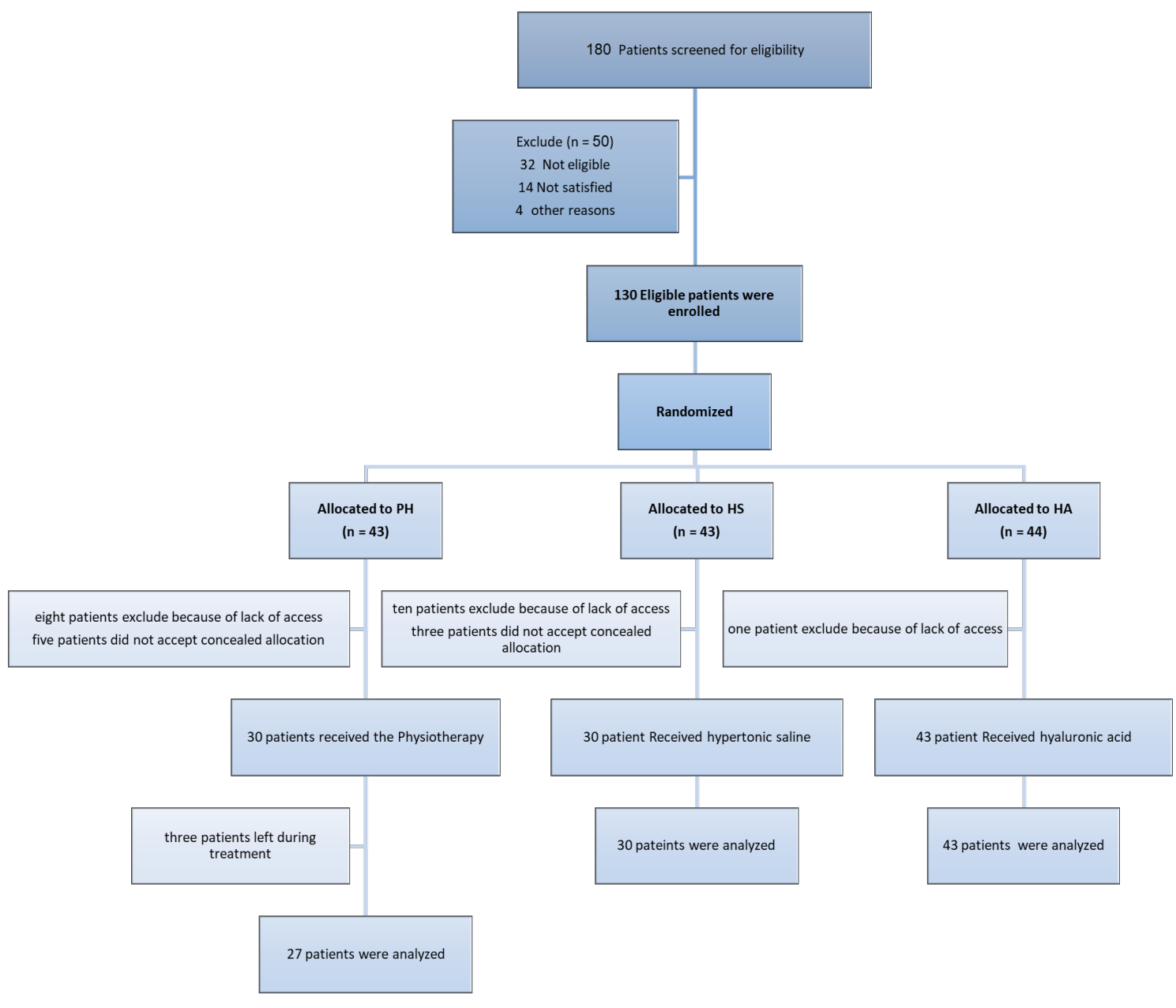

Figure 1.Flow diagram of the study population.

and sport/recreational subscales) were lower in the HS group, which tried to compensate through data analysis (table I).

\section{Clinical changes between treatment groups}

According to our findings, the two IA injections were proved to be effective in improving functional scores and reducing pain and symptoms in knee OA patients shortterm and long-term; furthermore, the $\mathrm{PH}$ group showed only significant pain reduction $(\mathrm{P}<0.001)$. Within five parts of the KOOS scale, the maximum amount of improvement for both the injected agents was detected in pain and sport/ recreational activities.

The comparative analysis of the injections and $\mathrm{PH}$ approved better scores for injections considering ADL, pain, and sport/recreational activities. Based on QOL scores, HS was significantly better than $\mathrm{PH}(\mathrm{P}<0.001)$, while HA was not $(\mathrm{P}=0.65)$. Comparing two injected agents, HS group obtained better scores not only in pain and symptom reduction, but also in function and QOL improvement (table II). Considering pain control through VAS, HS resulted in considerable pain reduction in comparison with $\mathrm{HA}$ and $\mathrm{PH}$ with a slight mean increase (from 6.1 to 6.9) between three to six months follow-up (figure 2).

Table 3 shows Stratified Analyses for the Radiographic knee severity With mean difference between-groups at 1,3 and 6 months follow up (MITT) (table III).

\section{Safety}

No significant complications, including infections (local or systemic), occurred in either of the two injection groups. 
Table I. Baseline characteristics of study participants.

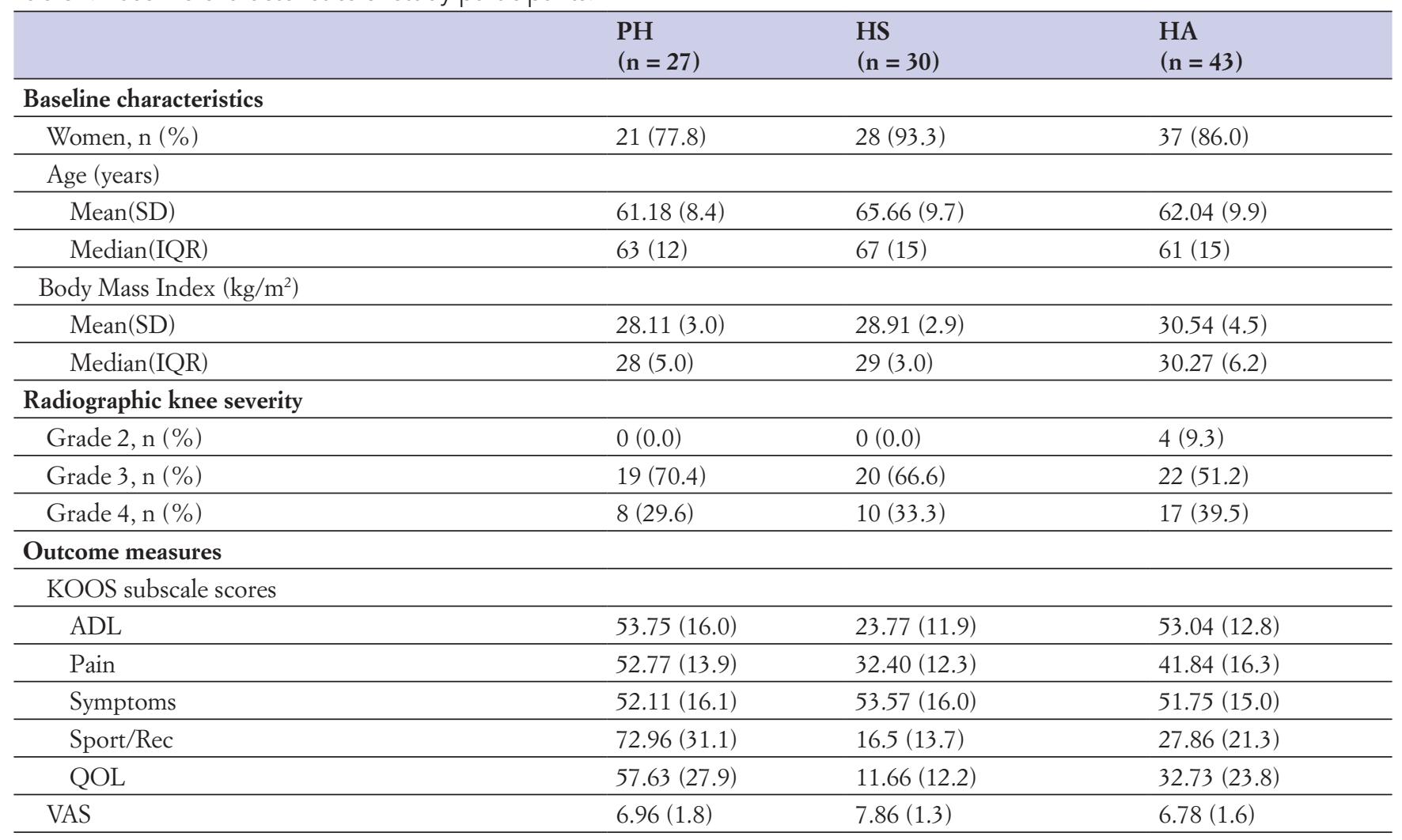

Variables are expressed as the Mean (standard deviation) and Median (interquartile range). Abbreviations: PH, Physiotherapy; HS, hypertonic saline; HA, hyaluronic acid; KOOS, Knee injury and Osteoarthritis Outcome Score ranging from 0 to 100 with high scores indicating fewer problems; VAS, visual analogue scales 0 to 10 .

Table II. Mean difference within-groups and difference between-groups at 1, 3 and 6 months follow up (MITT).

\section{Test of Within-group effects (mean change from baseline) \\ PH HS HA \\ Test of between-group effects (mean change from reference group)}

\begin{tabular}{|c|c|c|c|c|c|c|}
\hline Outcomes & Mean $(95 \% \mathrm{CI})$ & Mean $(95 \%$ CI $)$ & Mean $(95 \%$ CI $)$ & B (95\% CI) & $\beta(95 \% \mathrm{CI})$ & $\beta(95 \% \mathrm{CI})$ \\
\hline \multicolumn{7}{|l|}{$\mathrm{ADL}$} \\
\hline $\mathrm{T} 1$ & $-1.4(-6.0,3.2)$ & $32.1(28.1,36.2)^{\text {k.*k }}$ & $8.0(3.3,12.7)^{\text {with }}$ & $33.8(27.8,39.8)^{\text {nith }}$ & $9.3(2.8,15.9)^{* * x}$ & $24.8(18.8,30.8)^{\text {ntwit }}$ \\
\hline T3 & $0.02(-3.7,3.7)$ & $38.1(34.6,41.7)^{\text {i.k. }}$ & $14.2(10.6,17.8)^{n \times a k}$ & $38.4(33.3,43.5)^{n+x+a x}$ & $14.2(9.0,19.3)^{\text {santen }}$ & $24.4(19.4,29.3)^{*+x+k x}$ \\
\hline T6 & $-0.1(-3.8,3.6)$ & $40.1(35.9,44.4)^{\text {t.k.k }}$ & $17.5(13.1,22.0)^{n \star s / n}$ & $39.9(34.3,45,5)^{m, n+x}$ & $17.6(11.9,23.4)^{* * 2 x}$ & $22.4(16.2,28.6)^{n+k+x}$ \\
\hline \multicolumn{7}{|l|}{ Pain } \\
\hline $\mathrm{T} 1$ & $1.5(-3.4,6.5)$ & $32.1(27.5,36.8)^{k, k x}$ & $15.8(7.7,23.9)^{* x+x}$ & $30.9(24.1,37.6)^{n+w+4}$ & $14.4(4.9,23.8)^{n+s a t}$ & $16.5(7.3,25.7)^{\sin x}$ \\
\hline T3 & $5.3(-0.1,10.7)$ & $40.0(36.0,44.1)^{* * * x}$ & $21.8(14.2,29.4)^{k * x *}$ & $35.7(28.9,42.5)^{n+k+x+x}$ & $16.6(7.4,25.9)^{\text {knt }}$ & $19.1(10.4,27.7)^{\text {ththt }}$ \\
\hline T6 & $7.3(0.9,13.6)^{*}$ & $42.4(38.0,46.7)^{* . * m}$ & $21.9(14.0,29.9)^{k * x *}$ & $35.4(27.8,43.0)^{n+k+1+x}$ & $14.7(4.6,24.8)^{m+n}$ & $20.7(11.8,29.7)^{k n k t}$ \\
\hline \multicolumn{7}{|l|}{ Symptoms } \\
\hline $\mathrm{T} 1$ & $-2.2(-7.1,2.7)$ & $18.1(13.9,22.1)^{m+k x}$ & $6.1(1.6,10.7)^{n+n}$ & $20.8(14.8,26.8)^{*+\alpha+4}$ & $9.49(3.0,15.9)^{m+n}$ & $11.3(5.4,17.2)^{n+s+8}$ \\
\hline $\mathrm{T} 3$ & $2.2(-4.0,8.4)$ & $25.9(19.0,32.9)^{n+* x+k}$ & $9.9(5.2,14.7)^{n+x+8}$ & $22.4(12.9,31.8)^{\text {witat }}$ & $7.2(-0.5,14.9)$ & $15.2(6.6,23.8)^{\text {w.x }}$ \\
\hline T6 & $2.4(-3.2,8.1)$ & $28.2(21.2,35.3)^{\text {ntwat }}$ & $9.2(4.1,14.2)^{n+m+t h e}$ & $24.9(15.9,33.9)^{\text {ntant }}$ & $6.1(-1.3,13.6)$ & $18.8(10.1,27.5)^{k+k t h}$ \\
\hline
\end{tabular}

\section{HS vs $\mathrm{PH} \quad \mathrm{HA}$ vs $\mathrm{PH} \quad \mathrm{HS}$ vs HA}

$\beta(95 \% \mathrm{CI})$ 


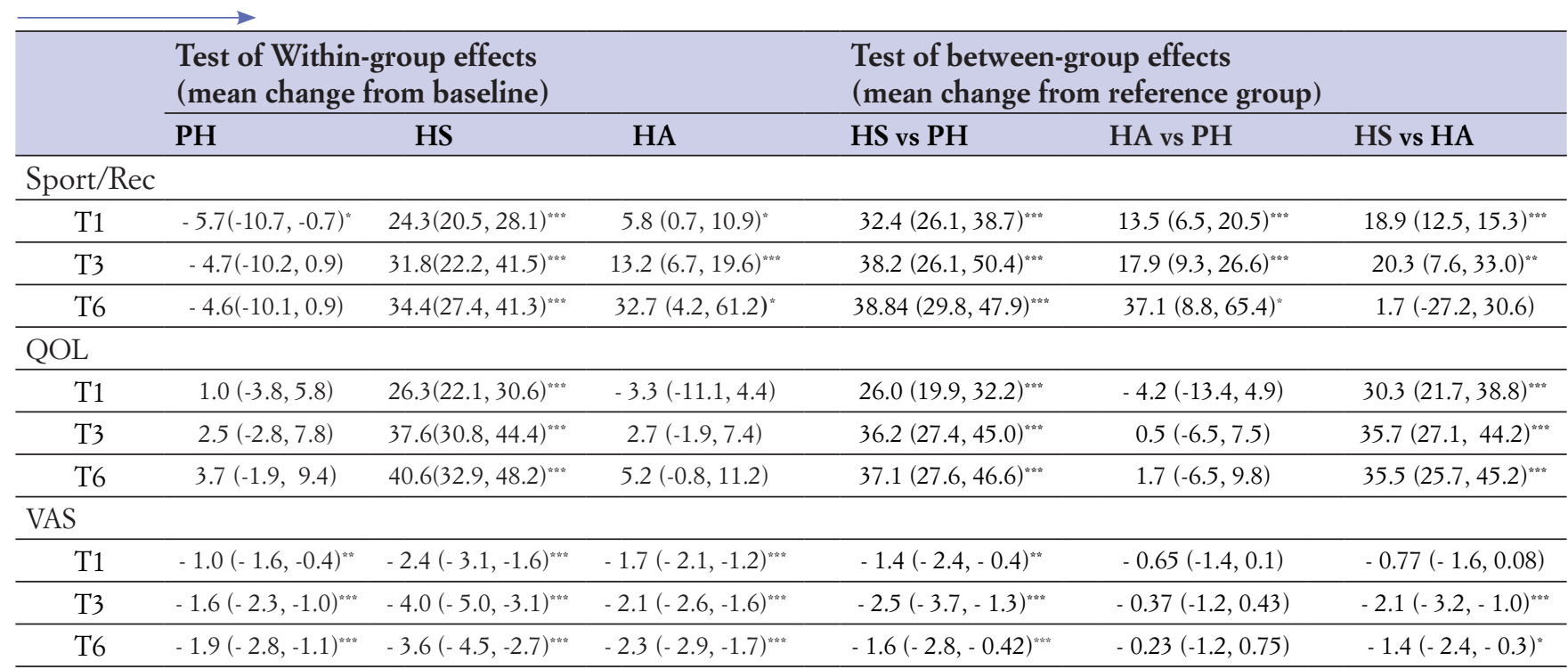

ADL: activities of daily living; QOLI: Quality of Life; VAS: visual analogue scales 0 to 10; T0: baseline data collection; T1: one-month data collection; T3: three-month data collection; T6: six-month data collection. a Adjusted generalized estimating equations model after controlling the baseline Outcome, sex, age, BMI. ***: P-value $<0.001 ; *$ : P-value $<0.01$. *: P-value $<0.05$

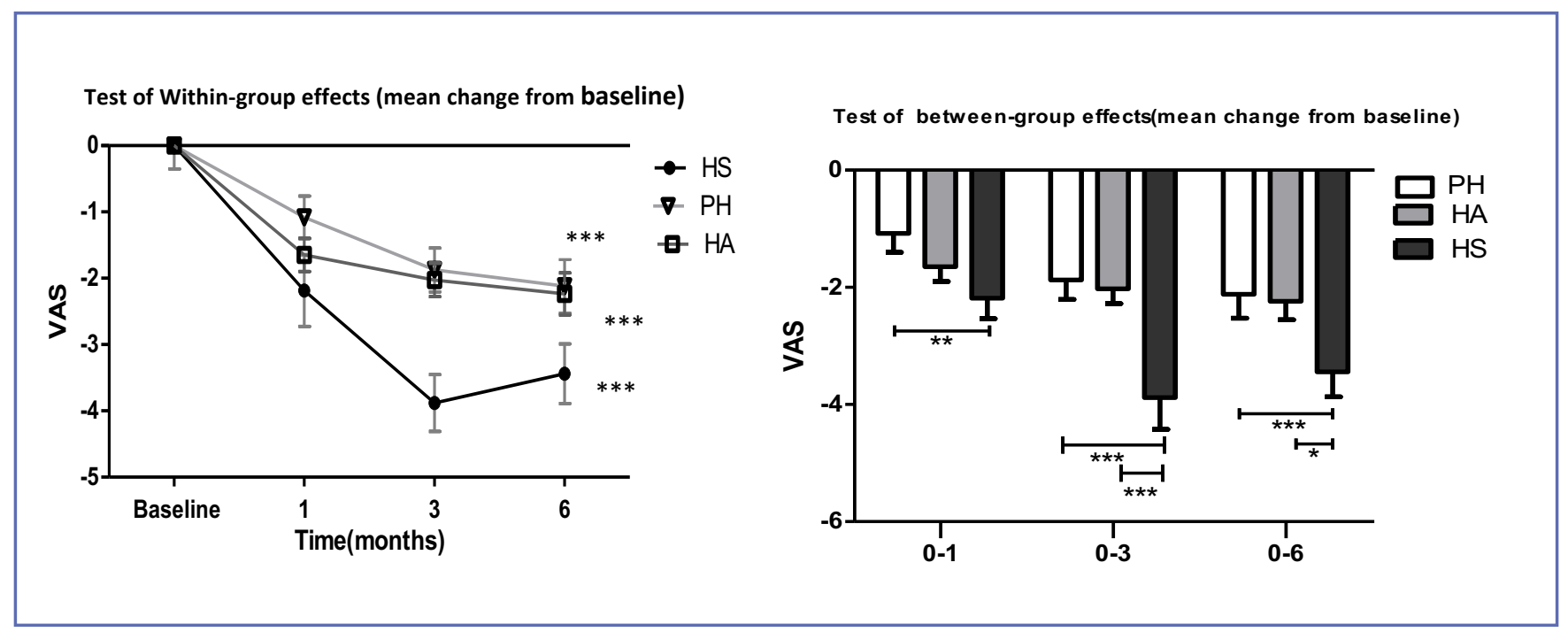

Figure 2. Pain changes in three groups based on VAS.

Acute flare reaction was detected in four patients (two in HA and two in HS), and they were not significantly different between the two groups (figure 3).

\section{DISCUSSION}

To the extent of our knowledge, for the first time, this study evaluated HS therapeutic effects through randomized clinical trial from the aspects of pain reduction, QOL, and functional improvements. Additionally, the safety evaluation of HS injection into the knee joint is of importance.
Our findings suggested $\mathrm{HS}$ as a proper treatment for knee OA, according to pain reduction, functional and QOL improvements observed in this study. Regarding symptom changes in HA and HS group, HS led to more improvement after six months compared to the first or third post-injection month. Symptoms in the KOOS questionnaire consist of locking, stiffness, and giving-away. It seems in long-term parallel to absorbing HA from joint and decreasing its effectiveness on symptoms, HS prolotherapy effects on degenerative changes in menisci and ligaments, yielded more complaint reduction of patients in HS group after six months. 
Table III. Stratified Analyses for the Radiographic knee severity With mean difference between-groups at 1, 3 and 6 months follow up (MITT).

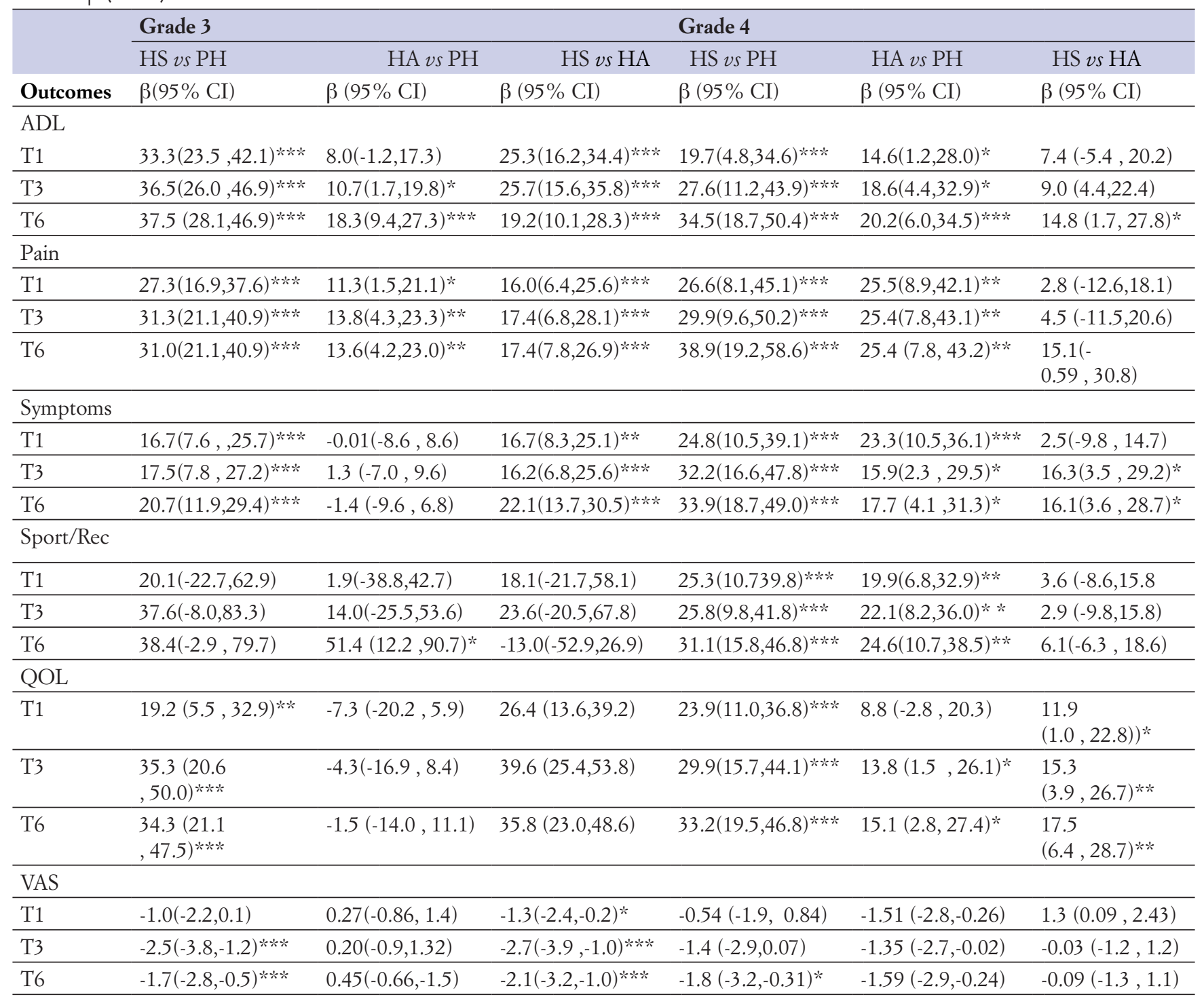

ADL: activities of daily living; QOLI: Quality of Life; VAS: visual analogue scales 0 to 10; T0: baseline data collection; T1: one-month data collection; T3: three-month data collection; T6: six-month data collection. ***: P-value $<0.001 ;{ }^{* *}$ : P-value $<0.01 ; *$ : P-value $<0.05$.

Because hypertonic dextrose prolotherapy influences periarticular components $(12,15,30,31)$, its long-term use is suggested to result in desirable outcomes, while there was no supporting study in the long term effect of hypertonic saline prolotherapy. Further studies are needed to evaluate ligamentous changes following HS injection is recommended. In this study, hypertonic saline and Hyaluronic acid treatment groups were significantly different in KOOS questionnaire subscales scores, with the superiority of hypertonic saline. In 2014, Arden et al. claimed that no remarkable difference exists between normal saline and hyaluronic acid in WOMAC questionnaire items (32). In 2018, Takamura et al. performed a study to compare the effects of normal saline and a high Molecular weight Hyalunoric acid using the WOMAC questionnaire; unlike the results of our study, there was a remarkable difference between two groups in pain and stiffness scale. The only statistically significant item between the two groups was physical function with better outcomes for Hyaluronic acid (33). These differences in findings could be interpreted with the hypertonic nature of the saline solution, which has been used in our study, which yielded better outcomes in knee OA patients. 


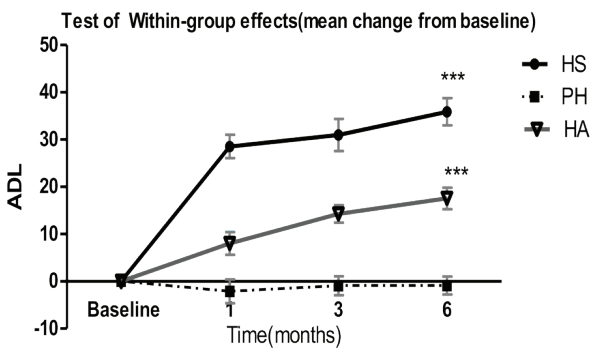

Test of between-group effects(mean change from baseline)
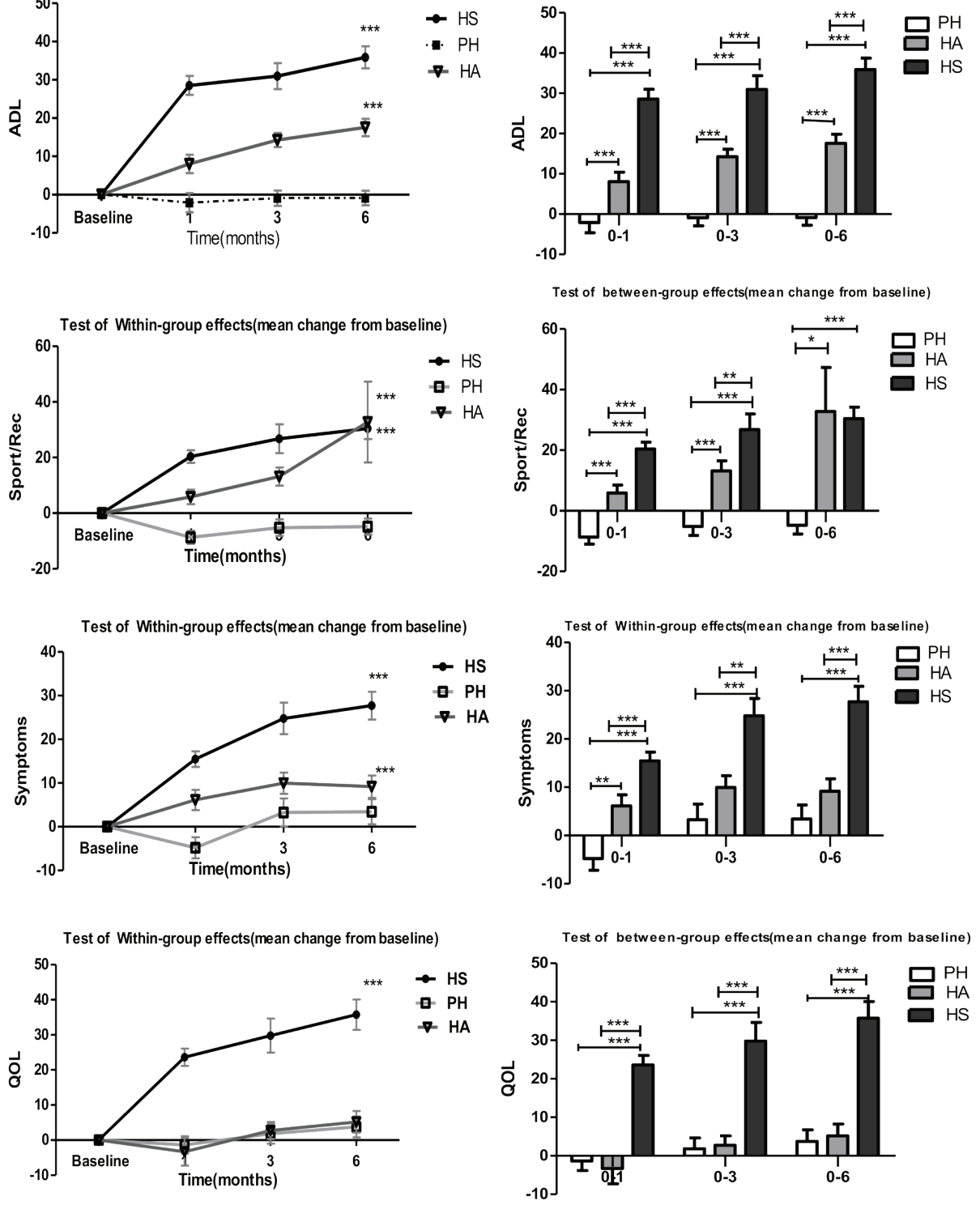

Test of Within-group effects(mean change from baseline)

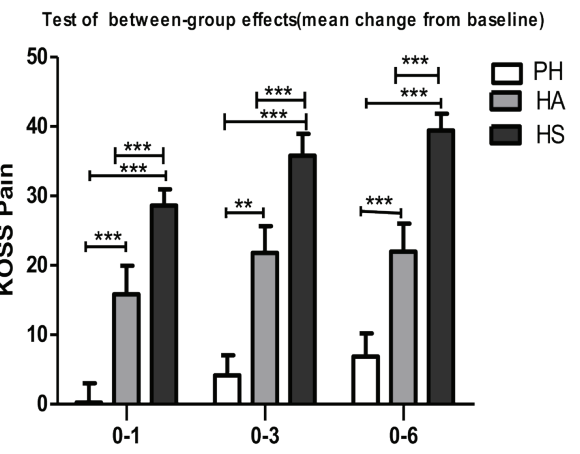

Figure 3. The KOOS subscales within group and between group effects. 
There are several studies on short-term and long-term effects of HA among knee OA patients on pain control and function improvements, in the same vein of our study (26, 34). In addition to subjective factors, other studies assessed objective measures consist of a decline in cytokines responsible for inflammation and radiologic findings to confirm the effectiveness of HA in knee OA (35-37). A recent multicenter study claimed HA injections delayed patient need for TKA (38). Moreover, symptom relief of saline was suggested in previous studies using saline as a placebo $(19,20)$. It is not surprising to detect only pain reduction in the $\mathrm{PH}$ group due to the use of pain relief modalities, including the US, TENS, and IR. Although these groups were educated on strengthening exercises, their adherence to exercise is questionable regarding the unsupervised program for exercises in this study (table IV).

Considering QOL, HS was better than $\mathrm{PH}$, while HA was not. Although this difference could be interpreted with differences in symptom relief and pain reduction, the number of visits for treatment seems to be important in the perception of the patient from QOL. Since the HS group received a single injection, they had better QOL scores comparing the HA group experienced three visits and $\mathrm{PH}$ group with 10 visits. Additionally, injected drug costs could be another reason from the perspective of patients.

This study had some limitations; first, we used subjective outcomes to evaluate the therapeutic effects of injected agents. Objective outcomes, including intra-articular inflammatory cytokines or imaging changes, were not investigated; second, baseline characteristics of patients in the HS group were different in some KOOS subscales; therefore, we attempted to use General Estimation Equation (GEE) indeed. Third, we did not assess patients' physical activity level or adherence to strengthening exercises (checklists were poorly completed) nor time from symptom onset; and eventually, we used HA for comparison group due to its approved short-term and long-term effects while other more common choices like corticosteroids have shortterm effectiveness. Studies with a larger sample size and longer follow-up with the evaluation of objective outcomes in addition to subjective outcomes are recommended.

\section{CONCLUSIONS}

Based on our findings, both HA and HS were efficacious based on pain, function, and QOL in knee OA patients with greater improvement in HS injected patients. It seems appropriate to introduce HS as an effective agent for knee OA treatment not only for short-term effects but also for the long-term. Eventually, HS prolotherapy is recommended in knee OA patients due to its safety and cost-effectiveness. The study meets the ethical standards of the journal (41).

\section{CONTRIBUTIONS}

Ensieh Taftain contributed to the design, guided the development of the study protocol, and participated in the physical examination. Sirous Azizi and Zahra Reza Soltani

Table IV. Studies comparing Saline with otherinjectable agents.

\begin{tabular}{|c|c|c|c|c|c|c|c|}
\hline Study & Type of study & $\begin{array}{l}\text { Year of } \\
\text { publication }\end{array}$ & $\begin{array}{l}\text { Number of } \\
\text { included } \\
\text { study/patient }\end{array}$ & Interventions & \multicolumn{2}{|c|}{ Duration of follow-up } & Conclusion \\
\hline Altman et al. & $\begin{array}{l}\text { Systematic } \\
\text { review \& } \\
\text { meta-analysis }\end{array}$ & 2016 & $38 />2500$ & $\begin{array}{l}\text { N/S vs HA, } \\
\text { CS , PRP }\end{array}$ & $<3$ months & 6-12 months & $\begin{array}{l}\text { Pain relief observed } \\
\text { with intra-articular } \\
\text { saline in addition to } \\
\text { other agents }\end{array}$ \\
\hline Bar-Or et al. & Review & 2017 & $73 / 5816$ & $\begin{array}{l}\text { Saline vs HA, } \\
\text { PRP, biologic }\end{array}$ & & & $\begin{array}{l}\text { Pain improvement with } \\
\text { Saline and other agents }\end{array}$ \\
\hline McCormack et al. & Clinical trial & 2017 & 368 & $\begin{array}{l}\mathrm{HA}+\mathrm{CS} v s \\
\mathrm{HA} \text { vs Saline }\end{array}$ & $\begin{array}{l}1,3 \\
6,12 \text { weeks }\end{array}$ & 18,26 weeks & $\begin{array}{l}\text { Greater pain } \\
\text { reductions with Cingal } \\
(\mathrm{HA}+\mathrm{CS})\end{array}$ \\
\hline
\end{tabular}

HA: hyaluronic acid; CS: corticosteroid; N/S: normal saline. 
contributed to the concept and literature review, coordinated and supervised the study. Afsaneh Dadarkhah and Sharif Najafi guided recruitment, performed the patient interviews and physical examination. Nastaran Maghbouli contributed to the design, and performed statistical analyses. Armin Khavandegar helped in draft the manuscript. All the authors participated in drafting and its final approval.

\section{REFERENCES}

1. Creamer P, Hochberg MC. Osteoarthritis of the knee. Lancet 1997;350(9087):1328.

2. Felson DT. The sources of pain in knee osteoarthritis. Curr Opin Rheumatol 2005;17(5):624-8.

3. Palazzo C, Ravaud J-F, Papelard A, Ravaud P, Poiraudeau $\mathrm{S}$. The burden of musculoskeletal conditions. PloS One 2014;9(3).

4. Salmon J, Rat A, Sellam J, et al. Economic impact of lowerlimb osteoarthritis worldwide: a systematic review of cost-ofillness studies. Osteoarthritis Cartilage 2016;24(9):1500-8.

5. Quintana JM, Arostegui I, Escobar A, Azkarate J, Goenaga JI, Lafuente I. Prevalence of knee and hip osteoarthritis and the appropriateness of joint replacement in an older population. Arch Intern Med 2008;168(14):1576-84.

6. Singh G, Triadafilopoulos G. Epidemiology of NSAID induced gastrointestinal complications. J Rheumatol Suppl 1999;56:18-24.

7. Davies NM, Jamali F. COX-2 selective inhibitors cardiac toxicity: getting to the heart of the matter. J Pharm Pharm Sci 2004;7(3):332-6.

8. Hassan F, Murrell WD, Refalo A, Maffulli N. Alternatives to biologics in management of knee osteoarthritis: a systematic review. Sports medicine and arthroscopy review. 2018;26(2):79-85.

9. Mora JC, Przkora R, Cruz-Almeida Y. Knee osteoarthritis: pathophysiology and current treatment modalities. J Pain Res 2018;11:2189.

10. Özgönenel L, Aytekin E, Durmuşoǧlu G. A double-blind trial of clinical effects of therapeutic ultrasound in knee osteoarthritis. Ultrasound Med Biol 2009;35(1):44-9.

11. Osiri M, Welch V, Brosseau L, et al. Transcutaneous electrical nerve stimulation for knee osteoarthritis. Cochrane Database Syst Rev 2000(4).

12. Hauser RA, Lackner JB, Steilen-Matias D, Harris DK. A systematic review of dextrose prolotherapy for chronic musculoskeletal pain. Clin Med Insights Arthritis Musculoskelet Disord 2016;9:CMAMD. S39160.

13. Distel LM, Best TM. Prolotherapy: a clinical review of its role in treating chronic musculoskeletal pain. PM\&R 2011;3:S78-S81.

14. Burdakov D, Jensen LT, Alexopoulos H, et al. Tandem-pore $\mathrm{K}+$ channels mediate inhibition of orexin neurons by glucose. Neuron 2006;50(5):711-22.

15. Jensen KT, Rabago DP, Best TM, Patterson JJ, Vanderby Jr R. Early inflammatory response of knee ligaments to prolotherapy in a rat model. J Orthop Res 2008;26(6):816-23.

\section{ACKNOWLEDGMENTS}

The authors would like to thank Dr. Shahram Rahimi who was involved in revising this article critically.

\section{CONFLICT OF INTERESTS}

The authors declare that they have no conflict of interests.

16. Rabago D, Best TM, Beamsley M, Patterson J. A systematic review of prolotherapy for chronic musculoskeletal pain. Clin J Sport Med 2005;15(5):E376.

17. Rabago D, Mundt M, Zgierska A, Grettie J. Hypertonic dextrose injection (prolotherapy) for knee osteoarthritis: Long term outcomes. Complement Ther Med 2015;23(3):388-95.

18. Bar-Or D, Rael LT, Brody EN. Use of saline as a placebo in intra-articular injections in osteoarthritis: potential contributions to nociceptive pain relief. Open Rheumatol J 2017;11:16.

19. Altman RD, Devji T, Bhandari M, Fierlinger A, Niazi F, Christensen R. Clinical benefit of intra-articular saline as a comparator in clinical trials of knee osteoarthritis treatments: A systematic review and meta-analysis of randomized trials. Semin Arthritis Rheum 2016;46(2):151-9.

20. Saltzman BM, Leroux T, Meyer MA, et al. The therapeutic effect of intra-articular normal saline injections for knee osteoarthritis: a meta-analysis of evidence level 1 studies. Am J Sports Med 2017;45(11):2647-53.

21. Halterman JA, Kwon HM, Wamhoff BR. Tonicity-independent regulation of the osmosensitive transcription factor TonEBP (NFAT5). Am J Physiol Cell Physiol 2012;302(1):C1-C8.

22. Yoon HJ, You S, Yoo SA, et al. NF-AT5 is a critical regulator of inflammatory arthritis. Arthritis Rheum 2011;63(7):1843-52.

23. Balazs EA, Watson D, Duff IF, Roseman S. Hyaluronic acid in synovial fluid. I. Molecular parameters of hyaluronic acid in normal and arthritic human fluids. Arthritis Rheum 1967;10(4):357-76.

24. Moreland LW. Intra-articular hyaluronan (hyaluronic acid) and hylans for the treatment of osteoarthritis: mechanisms of action. Arthritis Res Ther 2003;5(2):54.

25. Lo GH, LaValley M, McAlindon T, Felson DT. Intra-articular hyaluronic acid in treatment of knee osteoarthritis: a meta-analysis. Jama 2003;290(23):3115-21.

26. Bannuru R, Natov N, Dasi U, Schmid C, McAlindon T. Therapeutic trajectory following intra-articular hyaluronic acid injection in knee osteoarthritis-meta-analysis. Osteoarthritis Cartilage 2011;19(6):611-9.

27. Frizziero A, Vittadini F, Oliva F, et al. I.S.Mu.L.T. Hyaluronic acid injections in musculoskeletal disorders guidelines. Muscles Ligaments Tendons J 2018;8(3):364-98.

28. Rodriguez-Merchan EC. Intra-articular injections of hyaluronic acid and other drugs in the knee joint. HSS J 2013;9(2):180-2.

29. Barbieri E, Capparucci I, Mannello F, et al. Efficacy of a Treatment for Gonarthrosis Based on the Sequential Intra-Articu- 
lar Injection of Linear and Cross-Linked Hyaluronic Acids. Muscles Ligaments Tendons J 2019;9(4):606-14.

30. Collins N, Prinsen C, Christensen R, Bartels E, Terwee C, Roos E. Knee Injury and Osteoarthritis Outcome Score (KOOS): systematic review and meta-analysis of measurement properties. Osteoarthritis Cartilage 2016;24(8):1317-29.

31. Marot V, Murgier J, Carrozzo A, et al. Determination of normal KOOS and WOMAC values in a healthy population. Knee Surgery, Sports Traumatology, Arthroscopy 2019;27(2):541-8.

32. Rabago D, Nourani B. Prolotherapy for osteoarthritis and tendinopathy: a descriptive review. Curr Rheumatol Rep 2017;19(6):34.

33. Rezasoltani Z, Taheri M, Mofrad MK, Mohajerani SA. Periarticular dextrose prolotherapy instead of intra-articular injection for pain and functional improvement in knee osteoarthritis. J Pain Res 2017;10:1179.

34. Arden NK, Åkermark C, Andersson M, Todman MG, Altman RD. A randomized saline-controlled trial of NASHA hyaluronic acid for knee osteoarthritis. Curr Med Res Opin 2014;30(2):279-86.

35. Takamura J, Seo T, Strand V. A Pooled Analysis of Two Multicenter, Randomized Controlled Trials of a Single Intra-articular Injection of Gel-200 for Treatment of Osteoarthritis of the Knee. Clin Med Insights Arthritis Musculoskelet Disord 2018;11:1179544118773068.
36. Bannuru RR, Vaysbrot EE, Sullivan MC, McAlindon TE (Editors). Relative efficacy of hyaluronic acid in comparison with NSAIDs for knee osteoarthritis: a systematic review and meta-analysis. Seminars in arthritis and rheumatism. Elsevier 2014.

37. Gaston M, Tiemessen C, Philips J. Intra-articular hip viscosupplementation with synthetic hyaluronic acid for osteoarthritis: efficacy, safety and relation to pre-injection radiographs. Arch Orthop Trauma Surg 2007;127(10):899-903.

38. Vincent HK, Percival SS, Conrad BP, Seay AN, Montero C, Vincent KR. Hyaluronic acid (HA) viscosupplementation on synovial fluid inflammation in knee osteoarthritis: a pilot study. Open Orthop J 2013;7:378.

39. Nelson F, Zvirbulis R, Zonca B, et al. The effects of an oral preparation containing hyaluronic acid (Oralvisc $\AA$ ) on obese knee osteoarthritis patients determined by pain, function, bradykinin, leptin, inflammatory cytokines, and heavy water analyses. Rheumatol Int 2015;35(1):43-52.

40. Nicholls M, Shaw P, Niazi F, et al. Intra-articular hyaluronic acid delay to total knee by number of injection courses received: analysis of an administrative database. Osteoarthritis Cartilage 2020;28:S451.

41. Padulo J, Oliva F, Frizziero A, Maffulli N. Muscles, Ligaments and Tendons Journal - Basic principles and recommendations in clinical and field Science Research: 2018 update. Muscles Ligaments Tendons J 2018;8(3):305-7. 